\title{
Risk of cardiovascular disease among teachers in Cape Town: Findings of the South African PaCT pilot study
}

\author{
E C Laurence, ${ }^{1} \mathrm{MB}$ ChB, MSc (Clin Epidemiol); J Volmink, ${ }^{1,2}$ BSc, MB ChB, MPH, DPhil; T M Esterhuizen, ${ }^{1}$ MSc (Epidemiol); \\ S Dalal, ${ }^{3} \mathrm{PhD} ; \mathrm{M}$ D Holmes, ${ }^{3,4} \mathrm{MD}, \mathrm{DrPH}$ \\ ${ }^{1}$ Centre for Evidence-based Health Care, Faculty of Medicine and Health Sciences, Stellenbosch University, Tygerberg, Cape Town, South Africa \\ ${ }^{2}$ South African Cochrane Centre, South African Medical Research Council, Tygerberg, Cape Town, South Africa \\ ${ }^{3}$ Department of Epidemiology, Harvard School of Public Health, Boston, USA \\ ${ }^{4}$ Channing Division of Network Medicine, Department of Medicine, Brigham and Women's Hospital and Harvard Medical School, Boston, USA
}

Corresponding author: E C Laurence (carienl@sun.ac.za)

Background. The accelerating epidemic of cardiovascular disease (CVD) and other non-communicable diseases (NCDs) highlights the need to establish long-term cohort studies in Africa.

Objective. The Partnership for Cohort Research and Training (PaCT) seeks to study NCDs in South Africa (SA), Uganda, Tanzania and Nigeria on a long-term basis. Pilot studies at each site have tested feasibility. The SA site additionally studied the prevalence of CVD risk factors and categorised participants' 10 -year predicted risk of a cardiovascular event.

Methods. We enrolled teachers from 111 public schools in the Metro South Education District in Cape Town, SA, between January 2011 and May 2012. Participants completed a self-administered questionnaire and biological measurements, and chose post or email for 6-month follow-up.

Results. The participation of schools was permitted by $53.2 \%$ of principals, and 489 of 1779 teachers agreed to participate. Of teachers willing to participate in the follow-up, 52\% were retained, three-quarters by post and a quarter by email. Their mean age was 46.3 years and $70.3 \%$ were female. The prevalence of CVD risk factors was high and featured hypertension (48.5\%), hypercholesterolaemia (20.5\%), smoking (18.0\%), diabetes (10.1\%) and chronic kidney disease (10.4\%), while $84.7 \%$ were overweight or obese. Of the participants, $18.7 \%$ were at high risk of a heart attack or stroke within 10 years.

Conclusion. Establishing a cohort study among teachers has challenges but also opportunities for addressing CVD, which will soon impose a substantial burden on Cape Town's education system.

S Afr Med J 2016;106(10):996-1001. DOI:10.7196/SAMJ.2016.v106i10.10869

Cardiovascular disease (CVD) is the leading non-communicable disease (NCD) globally, contributing to 17 million deaths annually. ${ }^{[1]}$ According to the World Health Organization (WHO), more than 1.1 million deaths in the African region in 2005 were caused by CVDs and over half of these were caused by ischaemic heart disease and stroke. ${ }^{[2]}$ Projections suggest that this figure will almost double by 2030 and that ischaemic heart disease and stroke will overtake HIV as the leading cause of death in sub-Saharan Africa by 2030. ${ }^{[3]}$

The need to investigate and address the underlying causes of CVD in Africa has become urgent. Establishing observational cohort studies to study the biological, social, cultural and economic determinants of NCDs in low- and middle-income countries has been identified by the WHO as an important research priority. ${ }^{[1]}$ Yet data on NCDs in Africa are sparse, ${ }^{[4]}$ and there is currently great disparity in the number of adults enrolled in long-term cohorts in Africa compared with developed countries. ${ }^{[5]}$ This is problematic, as geographical, cultural, social and genetic differences between populations may limit the applicability of scientific findings from the North to the African continent. Adult health has been studied with success in other countries using professional cohorts such as the Nurses' Health Study, a teacher cohort in the EsMaestra study in Mexico $^{[6]}$ and the California Teachers Study. ${ }^{[7]}$

\section{Objective}

The Partnership for Cohort Research and Training (PaCT) has been initiated to study NCDs among a cohort of people drawn from various settings in four African countries (South Africa (SA),
Uganda, Tanzania and Nigeria) with the intention of following them over several years. To assess the feasibility of this approach, pilot studies were conducted at each of the sites involved. The SA pilot study, the findings from which are reported in this article, was conducted among teachers and aimed to: $(i)$ determine the prevalence and distribution of CVD risk factors and self-reported heart disease and stroke; (ii) determine the percentage of participants in three categories of 10-year predicted risk of a fatal or non-fatal cardiovascular event; and (iii) evaluate the feasibility of conducting a cohort study of NCDs among teachers.

\section{Methods}

\section{Design and setting}

The methods used in PaCT pilot studies are described in an overview article relating to the feasibility of a cohort study in sub-Saharan Africa. ${ }^{[8]}$ A baseline survey and 6-month longitudinal follow-up were conducted between January 2011 and May 2012 in the SA pilot. We aimed to recruit 1000 teachers aged 18 years and older from public schools in the urban area of the Metro South Education District (MSED) in Cape Town, SA. The MSED contains 4166 teachers employed at 189 schools, representing different socioeconomic levels. To improve recruitment, we selected schools where $\geq 20$ teachers were employed, resulting in a total of 111 schools with approximately 3166 teachers. We first requested written permission from the principals and then sent questionnaire-consent packages for distribution at the schools. Participants received feedback about their blood pressure and laboratory results by personalised SMS (text 
message) or email and were advised to see their regular doctor for readings outside the normal range. Communication with schools was primarily through telephone and email, to assess whether these methods could be used effectively in a countrywide cohort.

\section{Data collection}

Willing teachers enrolled by completing the consent forms and a 1-hour-long baseline questionnaire, and returned these in prepaid envelopes to the study co-ordination centre. Trained nurses visited schools at prearranged times to collect physical measurements (blood pressure, pulse rate, height, weight and waist circumference) and non-fasting biological samples (blood and urine) from participants. Details about data collection and measurements are summarised in Appendix 1. Willing participants received a follow-up questionnaire by email or post, according to their preference.

A personal history of heart disease or stroke was self-reported; a positive family history referred to first-degree relatives with coronary heart disease (CHD) or stroke. Being a current smoker was defined as self-reported use of tobacco products at the time of assessment. Hypertension was defined as having a systolic blood pressure $\geq 140 \mathrm{mmHg}$ or a diastolic blood pressure $\geq 90 \mathrm{mmHg}$ or being on antihypertensive treatment. Diabetes was defined as having a non-fasting blood glucose level $\geq 11.1 \mathrm{mmol} / \mathrm{L}$ or being on hypoglycaemic treatment. A desirable total cholesterol (TC) level was defined as $<5.18 \mathrm{mmol} / \mathrm{L}$, borderline high TC as $5.18-6.21 \mathrm{mmol} / \mathrm{L}$, high TC as $6.22-7.99 \mathrm{mmol} / \mathrm{L}$ and extremely high $\mathrm{TC}$ as $\geq 8 \mathrm{mmol} / \mathrm{L} \cdot{ }^{\left[{ }^{9]}\right.}$ Chronic kidney disease (CKD) was defined as having proteinuria $\geq 0.30 \mathrm{mg} / \mathrm{mg}$ or a glomerular filtration rate (GFR) $<60 \mathrm{~mL} / \mathrm{min} / 1.73 \mathrm{~m}^{2}$, calculated using the re-expressed Modification of Diet in Renal Disease (MDRD) study equation, or self-reported structural abnormalities of the kidneys. A body mass index (BMI) of $25.0-29.9 \mathrm{~kg} / \mathrm{m}^{2}$ was classified as being overweight and a BMI of $\geq 30 \mathrm{~kg} / \mathrm{m}^{2}$ as being obese. Abdominal obesity was defined using the National Cholesterol Education Program Third Adult Treatment Panel (NCEP-ATP III) recommendations (waist circumference $\geq 102 \mathrm{~cm}$ for males and $\geq 88 \mathrm{~cm}$ for females) and the International Diabetes Federation (IDF) recommendations (waist circumference $\geq 94 \mathrm{~cm}$ for males and $\geq 80 \mathrm{~cm}$ for females).

\section{Ethical considerations}

Ethical approval for the South African $\mathrm{PaCT}$ pilot study was obtained from the Health Research Ethics Committee of the Faculty of Medicine and Health Sciences at
Stellenbosch University (ref. no. N10/05/172) and the Harvard School of Public Health (HSPH) Institutional Review Board (ref. no. 19431-101). The Western Cape Education Department approved the study (ref. no. 20100426-0012) and the relevant teacher trade unions were informed about the study through a letter sent to their provincial offices.

\section{Analysis}

\section{Cardiovascular risk prediction}

Participants were categorised as at high risk ( $\geq 20.0 \%)$, intermediate risk (10.0 - 19.9\%) or low risk $(0-9.9 \%)$ for heart attack or stroke in the following 10 years, using the WHO and International Society of Hypertension (WHO/ISH) cardiovascular risk prediction charts for the WHO epidemiological subregion AFR E (SA).${ }^{[10]}$ Categorisation is based on five risk factors: age, current smoking status, systolic blood pressure, TC and presence or absence of diabetes mellitus. In addition, participants with established CVD (self-reported CHD or stroke), very high levels of individual risk factors (TC $\geq 8 \mathrm{mmol} / \mathrm{L}$, or TC/high-density lipoprotein cholesterol (TC/HDL-C) ratio $>8$, or a blood pressure $>160 / 100 \mathrm{mmHg}$ ) or $\mathrm{CKD}$ were categorised as having high cardiovascular risk. In a post-hoc analysis, the impact of BMI on CVD risk was assessed using the non-laboratory-based Framingham 10-year risk calculator. ${ }^{[11]}$

\section{Statistical analysis}

Statistical analysis of the data was performed using STATA IC 10 statistical software
(StataCorp, USA). Continuous variables were summarised as means (standard deviation (SD)) for normally distributed data and medians (interquartile range (IQR)) for asymmetrical data. Categorical variables were summarised as frequencies and percentages. Independent-sample $t$-tests were used to compare means of normally distributed continuous variables between groups, while $\chi^{2}$ tests were used to compare proportions. Where cardiovascular risk factors were compared between men and women, $p$-values were derived from Wald $\chi^{2}$ tests using multivariate binary logistic regression, adjusting for age and clustering by school. Predicted cardiovascular risk estimates are reported as proportions and 95\% confidence intervals (CIs) and were adjusted for clustering by school.

\section{Results \\ Baseline study sample and characteristics}

The baseline sample consisted of 489 participants, of whom $70.3 \%$ were females. The mean age of participants at baseline was 46.3 years (SD 8.5). Cardiovascular risk could not be determined for 12 male and 23 female participants, including 3 of unknown age, 19 who had no blood pressure readings and 13 with unknown diabetic status. Therefore 454 participants were included in the analysis.

Baseline characteristics summarised by gender are shown in Table 1 . With the exception of age $(p=0.733)$ and blood glucose $(p=0.659)$, significant differences were
Table 1. Baseline characteristics stratified by gender

\begin{tabular}{|c|c|c|c|}
\hline Characteristic & $\begin{array}{l}\text { Male } \\
(N=133)\end{array}$ & $\begin{array}{l}\text { Female } \\
(N=321)\end{array}$ & $p$-value \\
\hline Age (yr), mean (SD) & $46.8(9.1)$ & $46.5(8.0)$ & 0.733 \\
\hline Current smoking $(n, \%)$ & $31(24.4)$ & $45(15.2)$ & 0.042 \\
\hline Systolic blood pressure (mmHg), mean (SD) & $140.2(17.9)$ & $131.8(18.3)$ & $<0.001$ \\
\hline Diastolic blood pressure $(\mathrm{mmHg})$, mean $(\mathrm{SD})$ & $81.6(10.6)$ & $78.3(10.8)$ & 0.004 \\
\hline Blood glucose (mmol/L), median (IQR) & $5.2(4.7-5.8)$ & $5.2(4.7-5.8)$ & 0.659 \\
\hline $\mathrm{TC}(\mathrm{mmol} / \mathrm{L})$, mean $(\mathrm{SD})$ & $5.5(0.99)$ & $5.3(1.1)$ & 0.031 \\
\hline HDL-C (mmol/L), mean (SD) & $1.2(0.3)$ & $1.5(0.4)$ & $<0.001$ \\
\hline Serum creatinine $(\mu \mathrm{mol} / \mathrm{L})$, median $(\mathrm{IQR})$ & $90(80-99)$ & $66(59-74)$ & $<0.001$ \\
\hline $\begin{array}{l}\text { Urinary protein creatinine ratio }(\mathrm{mg} / \mathrm{mg}) \text {, } \\
\text { median (IQR) }\end{array}$ & $0.06(0.05-0.07)$ & $0.08(0.06-0.11)$ & $<0.001$ \\
\hline $\mathrm{GFR}^{\star}\left(\mathrm{mL} / \mathrm{min} / 1.73 \mathrm{~m}^{2}\right)$, mean $(\mathrm{SD})$ & $80.8(15.1)$ & $85.0(17.1)$ & 0.011 \\
\hline Height (m), mean (SD) & $1.73(0.08)$ & $1.58(0.06)$ & $<0.001$ \\
\hline Weight $(\mathrm{kg})$, mean $(\mathrm{SD})$ & $87.8(17.7)$ & $81.2(18.4)$ & $<0.001$ \\
\hline BMI $\left(\mathrm{kg} / \mathrm{m}^{2}\right)$, mean $(\mathrm{SD})$ & $29.1(4.8)$ & $32.4(7.0)$ & $<0.001$ \\
\hline Waist circumference $(\mathrm{cm})$, mean $(\mathrm{SD})$ & $97.6(12.7)$ & $94.6(13.8)$ & 0.029 \\
\hline
\end{tabular}


observed between males and females. More males indicated that they were current smokers $(p=0.042$ ), with the highest proportion of smokers being in the age category 50 - 59.9 years for both genders. The average systolic and diastolic blood pressure for males was higher than for females $(p<0.001$ and $p=0.004$, respectively). The mean BMI for males was in the overweight category and for females was in the obese category $(p<0.001)$.

\section{Prevalence of CVD risk factors}

Cardiovascular risk factors are shown for the total population, and stratified by gender (Table 2). Of the total population, $48.5 \%$ (220/454) had hypertension. Less than half of these participants (106/220, $48.2 \%)$ reported being on antihypertensive treatment and only half of those who were on medication had readings within the normal range. Of the participants, $10.1 \%(46 / 454)$ had diabetes and a further $3.3 \%(15 / 454)$ were at an increased risk of diabetes, based on having a random plasma glucose level between 7.8 and $11.0 \mathrm{mmol} / \mathrm{L}$. Less than half $(44.6 \%)$ of the participants had random TC levels within the normal range. Males had a higher prevalence of smoking $(p=0.042)$, hypertension based on blood pressure readings $(p=0.001)$, and TC/HDL-C ratio $>8$ $(p=0.031)$, while more females were obese $(p=0.007)$ and abdominally obese $(p<0.001)$.

\section{Obesity and family history}

Only $15.2 \%$ of participants had a BMI within the normal range, while $31.1 \%$ of participants had a BMI in the overweight range and a further $53.6 \%$ had a BMI in the obese range. According to the NCEP-ATP III and IDF recommendations, $61.2 \%$ and $78.4 \%$ of participants, respectively, were abdominally obese. Of the participants, $41.6 \%$ reported a family history of CHD or stroke among first-degree relatives, with similar rates for males and females.

\section{Calculated CVD risk}

The risk of suffering a CVD event over the following 10 years, according to the WHO/ISH risk prediction charts and the Framingham risk calculator using BMI, is shown in Table 3. Based on the WHO/ISH risk prediction charts, 79.7\% (95\% CI 74.9 - 84.6\%) of participants were at low cardiovascular risk, $1.6 \%$ (95\% CI $0.1-3.0 \%)$ were at intermediate risk and 18.7\% (95\% CI 14.2 - 23.1\%) were at high risk. Of these, only 3 participants were categorised at high risk based on the charts alone. A further 82 participants were categorised at high risk either because of established CVD (selfreported CHD or stroke), very high levels of

Table 2. Distribution of cardiovascular risk factors by gender

\begin{tabular}{|c|c|c|c|c|}
\hline Cardiovascular risk factor & $\begin{array}{l}\text { Total } \\
(N=454) \\
n(\%)\end{array}$ & $\begin{array}{l}\text { Male } \\
(N=133) \\
n(\%)\end{array}$ & $\begin{array}{l}\text { Female } \\
(N=321) \\
n(\%)\end{array}$ & $p$-value ${ }^{\star}$ \\
\hline Current smoker $(N=423)$ & $76(18.0)$ & $31(24.4)$ & $45(15.2)$ & 0.042 \\
\hline Hypertension $^{\dagger}$ & $220(48.5)$ & $72(54.1)$ & $148(46.1)$ & 0.121 \\
\hline Blood pressure $\geq 140 / 90 \mathrm{mmHg}$ & $166(36.6)$ & $63(47.4)$ & $103(32.1)$ & 0.001 \\
\hline Blood pressure $\geq 160 / 100 \mathrm{mmHg}$ & $42(9.3)$ & $15(11.3)$ & $27(8.4)$ & 0.379 \\
\hline Diabetes $^{\ddagger}$ & $46(10.1)$ & $17(12.8)$ & $29(9.0)$ & 0.167 \\
\hline Glucose $7.8-11.0 \mathrm{mmol} / \mathrm{L}$ & $15(3.3)$ & $2(1.5)$ & $13(4.1)$ & 0.148 \\
\hline \multicolumn{5}{|l|}{$\mathrm{TC}^{\S}(N=448)$} \\
\hline Borderline high & $156(34.8)$ & $51(38.4)$ & $105(33.3)$ & 0.340 \\
\hline High & $87(19.4)$ & $31(23.3)$ & $56(17.8)$ & 0.281 \\
\hline Extremely high & $5(1.1)$ & $1(0.8)$ & $4(1.3)$ & 0.484 \\
\hline $\mathrm{TC} / \mathrm{HDL}-\mathrm{C}$ ratio $>8$ & $4(0.9)$ & $3(2.3)$ & $1(0.3)$ & 0.031 \\
\hline $\begin{array}{l}\text { Personal history of CHD } / \text { stroke } \\
(N=445)\end{array}$ & $19(4.3)$ & $9(6.9)$ & $10(3.2)$ & 0.054 \\
\hline $\begin{array}{l}\text { Family history of CHD/stroke } \\
(N=445)\end{array}$ & $185(41.6)$ & $53(40.5)$ & $132(42.0)$ & 0.782 \\
\hline $\mathrm{CKD}^{* *}$ & $47(10.4)$ & $12(9.0)$ & $35(10.9)$ & 0.559 \\
\hline Overweight $^{\dagger \dagger}$ & $141(31.1)$ & $56(42.4)$ & $85(26.5)$ & 0.811 \\
\hline Obese $^{\dagger \dagger}$ & $243(53.6)$ & $49(37.1)$ & $194(60.4)$ & 0.007 \\
\hline Abdominal obesity (NCEP-ATPIII) ${ }^{\ddagger}$ & $277(61.2)$ & $54(40.6)$ & $223(69.7)$ & $<0.001$ \\
\hline Abdominal obesity (IDF) $)^{\varsigma \varsigma}$ & $355(78.4)$ & $81(60.9)$ & $274(85.6)$ & $<0.001$ \\
\hline \multicolumn{5}{|c|}{ 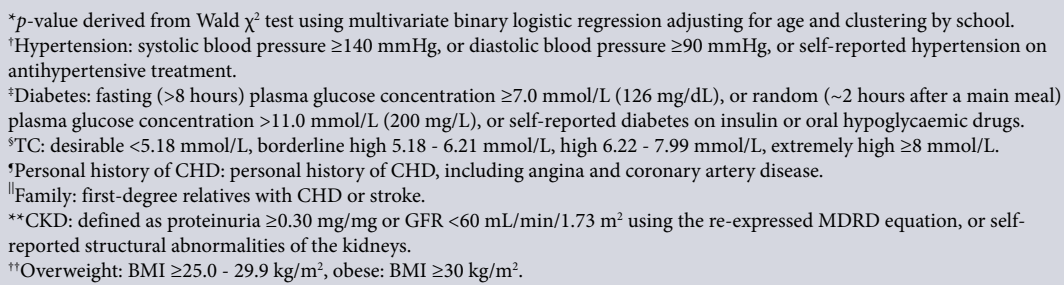 } \\
\hline \multicolumn{5}{|c|}{ FNCEP-ATP-III definition for abdominal obesity: male $\geq 102 \mathrm{~cm}$, female $\geq 88 \mathrm{~cm}$. } \\
\hline
\end{tabular}

Table 3. The 10-year predicted CVD risk using the WHO/ISH charts and the Framingham risk calculator

\begin{tabular}{|c|c|c|}
\hline 10-year predicted CVD risk & $\begin{array}{l}\text { WHO/ISH risk chart* } \\
(N=454) \\
n \text { (cluster-adjusted \%, } \\
95 \% \mathrm{CI})\end{array}$ & $\begin{array}{l}\text { Framingham risk calculator using } \\
\mathrm{BMI}^{\dagger} \\
(N=454) \\
n \text { (cluster-adjusted } \%, 95 \% \mathrm{CI})\end{array}$ \\
\hline Low $(0-9.9 \%)$ & $362(79.7,74.9-84.6)$ & $293(64.6,59.7-69.4)$ \\
\hline Intermediate (10 - 19.9\%) & $7(1.6,0.1-3.0)$ & $97(21.4,18.0-24.9)$ \\
\hline $\operatorname{High}(\geq 20 \%)$ & $85(18.7,14.2-23.1)$ & $63(13.9,9.9-18.0)$ \\
\hline \multicolumn{3}{|c|}{$\begin{array}{l}{ }^{*} \text { The WHO/ISH charts incorporate gender, age, smoking status, systolic blood pressure, TC and diabetes. In addition, } \\
\text { established CVD (CHD/stroke), CKD and extremely high levels of cholesterol and blood pressure were categorised as high risk } \\
\text { 'The Framingham risk calculator incorporates gender, age, smoking status, systolic blood pressure, treatment for hypertension } \\
\text { and diabetes, and BMI. }\end{array}$} \\
\hline
\end{tabular}

individual risk factors (TC $\geq 8 \mathrm{mmol} / \mathrm{L}$, or $\mathrm{TC} /$ HDL-C ratio $>8$, or blood pressure $>160 / 100$ $\mathrm{mmHg}$ ) or CKD.

The Framingham risk calculator identified more participants at intermediate risk $(21.4 \%, 95 \%$ CI $18.0-24.9 \%)$ probably because of the important role of overweight and obesity. However, it categorised fewer participants at high risk (13.9\%, 95\% CI 9.9 $18.0 \%$ because it does not take CKD or extreme cholesterol and blood pressure levels into account. 


\section{Baseline: 111 school principals invited to participate}

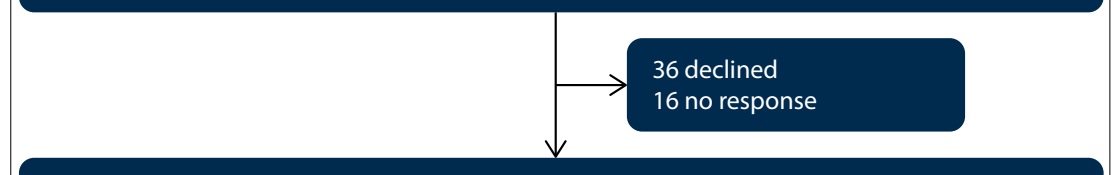

59 principals gave written permission to approach their teachers

1779 document packs delivered to participating schools

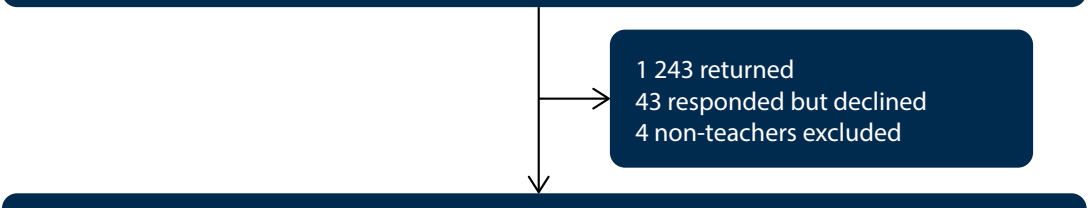

489 teachers enrolled in the study

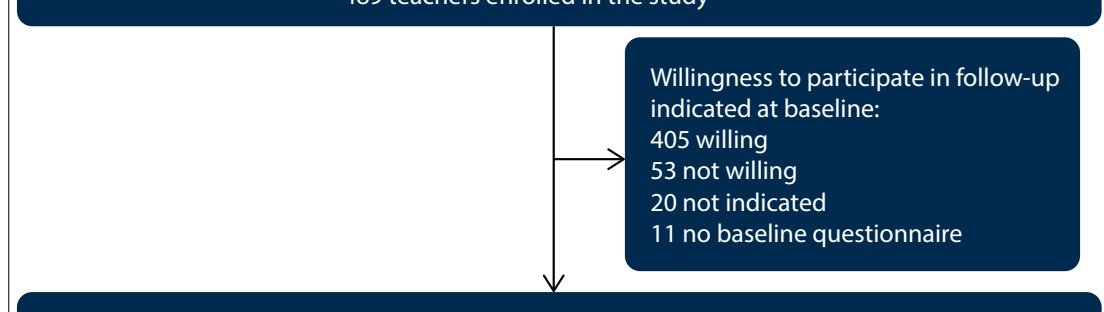

Follow-up after 6 months: 221/425 participants

Fig. 1. Recruitment at baseline and retention after 6 months.

Table 4. Prevalence of risk factors for CVD in the SA PaCT pilot study compared with crude adjusted 2008 estimates reported for SA in the WHO Global Status Report on NCDs ${ }^{[13]}$

\begin{tabular}{lll}
\hline CVD risk factor & $\begin{array}{l}\text { SA PaCT pilot study, } \\
\text { \% (95\% CI) }\end{array}$ & $\begin{array}{l}\text { Global Status Report on NCDs, } \\
\text { \% (95\% CI) }\end{array}$ \\
\hline Smoking & $17.9(14.3-21.6)$ & $14.0(11.7-16.3)$ \\
Raised TC & $55.4(50.7-59.9)$ & $34.0(20.7-49.2)$ \\
Overweight and obesity & $84.8(81.4-88.1)$ & $65.4(61.8-68.5)$ \\
Hypertension & $48.5(43.8-53.1)$ & $42.2(38.4-46.2)$ \\
Diabetes & $10.1(7.3-12.9)$ & $10.6(7.0-14.8)$
\end{tabular}

\section{Cohort feasibility}

Results from recruitment at baseline and retention after 6 months are summarised in Fig. 1. Following three rounds of invitation, 53.2\% (59/111) principals agreed to the participation of their school, $32.4 \%$ (36/111) declined either without reason or reported a lack of time or interest from the teachers, and $14.4 \%(16 / 111)$ did not reply following several reminders. Based on the number of teachers employed, 1779 questionnaire-consent packages were delivered to participating schools. A total of 489 teachers enrolled in the study and 478 of these completed a baseline questionnaire. Participation was better at the schools where the principals themselves enrolled, with $42.2 \%$ (444/1 053) document packages returned from their schools compared with only $6.2 \%(45 / 726)$ from schools where the principal chose not to enrol. We also found that more primary school principals agreed to participate (39/67 schools, 58.2\%) compared with secondary school principals (20/44 schools, $45.5 \%)$, and more primary school teachers enrolled (328/1121, 29.2\%) compared with secondary school teachers (161/658, 24.5\%).

Cardiovascular risk could not be determined for 35 participants in our analyses because of missing data. Questions relating to infectious conditions and mental health had more missing responses, with 41 and 149 participants choosing not to answer questions relating to TB and HIV, respectively, and 90 participants with missing mental health data. ${ }^{[12]}$ This may be explained by the sensitive nature of these questions. Across all sites, a strong number of participants indicated a willingness to provide biological samples and $93 \%$ of SA participants provided blood samples.

At the 6-month follow-up, assessment questionnaires were sent to 425 participants who had indicated at baseline that they were willing to participate in the follow-up, and $52.0 \%(221 / 425)$ of these were returned. Posted questionnaires were returned by $75.0 \%$, compared with $25.0 \%$ who responded through email.

\section{Discussion \\ CVD risk factors and calculated CVD risk}

Teachers in the MSED in Cape Town represent a group at high risk of chronic NCDs, in particular CVD. In this study, $18.7 \%$ of 454 participants were at a high risk of developing a heart attack or stroke during the next 10 years, requiring intensive lifestyle interventions and appropriate drug therapy. The Framingham risk score that incorporates BMI identified more participants at intermediate risk but fewer participants at high risk compared with the WHO/ISH charts. ${ }^{[10,11]}$ It is therefore important to incorporate BMI into risk calculators for SA as well as taking CKD and extreme cholesterol and blood pressure levels into account.

With the exception of diabetes, the SA $\mathrm{PaCT}$ pilot study population had a higher prevalence for each individual risk factor than the crude adjusted estimates for South Africans reported in the WHO Global Status Report on NCDs for 2008 ${ }^{[13]}$ (Table 4). The prevalence of hypertension in this pilot was lower $(48.5 \%)$ than that reported in the PURE NCD risk factor study for SA participants (57.1\%). ${ }^{[14]}$ Rates of overweight, obesity and central obesity were high in our study. In the NHANES-1 study, $43.0 \%$ of males and $62.4 \%$ of females in Western Cape Province had a BMI $>25 \mathrm{~kg} / \mathrm{m}^{2},{ }^{[15]}$ compared with $79.3 \%$ and $86.9 \%$, respectively, found in our study. This could be explained by the urban location of our pilot, with teachers having better access to food and less free time to exercise.

\section{Cohort feasibility}

Teachers represent an ideal group for studying the aetiology of NCDs through longitudinal studies because of their large numbers, gender and ethnic diversity, range of exposures and the ease with which they can be located. However, recruitment and retention among teachers has unique challenges, such as long interruptions in recruitment because of school holidays. The low response from principals (53.2\%) and teachers at baseline (489/1 779 documents 
returned), as well as low retention after 6-month follow-up (52.0\%) indicates that our methods to recruit and retain teachers into a large cohort study need improvement. Further pilot studies are necessary to test the best methods of recruiting large numbers of participants, keeping in mind the need for recruitment methods to be sufficiently simple to be scalable.

The majority of participants (91.0\%) were from schools where the principals themselves enrolled, reflecting the importance of gaining principals' support and enrolling them first. Alternatively, efficient methods of enrolling teachers directly, as done in the esMaestra study in Mexico ${ }^{[6]}$ and the California Teachers Study, ${ }^{[7]}$ could be tried. Lower participation rates at secondary schools (24.5\%) than primary schools $(29.2 \%)$ may be explained by secondary school teachers having less spare time. Participation could be improved by raising awareness about chronic NCDs and the importance of research before recruitment starts, meeting with the principals directly, simplifying and shortening the questionnaires and focusing on primary school teachers. To prevent loss of documents through the postal system and to improve completeness of information, data should be collected through an interviewer or obtained online.

The PaCT pilot studies conducted in Uganda and Nigeria reached their target sample size at enrolment and showed the highest retention rates at 6-month follow-up, ranging between $85.0 \%$ and $96.0 \%$. In Nigeria, nurses were enrolled and in Uganda participants were enrolled from rural and peri-urban communities and data collected through face-to-face interview, whereas in SA and Tanzania teachers were enrolled and data collected by self-administered questionnaires returned by post. ${ }^{[8]}$ Better response rates in Nigeria and Uganda indicate that building rapport through face-to-face interviews may be a better approach than self-administered questionnaires and that community-based populations or healthcare professionals such as nurses represent a good alternative study population.

\section{Study limitations}

Bias may have been introduced in our study through self-selection of participants with NCDs leading to overestimation of the prevalence of NCDs and risk factors. However, we think this is unlikely. We limited the impact of absenteeism from work on our study by arranging repeat visits to schools; however, if teachers with NCDs were more likely not to respond, our results may have resulted in an underestimation of the true prevalence of NCDs. Considering that NCDs are often asymptomatic until complications set in, it is unlikely that knowledge of pre-existing conditions among participants would have had a substantial impact on their participation.

A further limitation of our study is that the WHO/ISH risk prediction charts that we employed are designed for use in clinical practice to detect people aged $\geq 40$ years at high risk for CVD who qualify for treatment. The Framingham risk score was purely used to assess the impact of BMI on CVD risk, even though it has not been validated in SA. Our study population had high rates of overweight and obesity and was relatively young, with almost one-fifth aged $<40$ years. In younger populations, it is preferable to determine lifetime risk or to report the relative risk compared with people with ideal risk factor levels. Currently there are no risk prediction calculators that incorporate BMI to predict risk over periods $>10$ years that have been validated in African countries, underscoring the importance of establishing large cohort studies to collect the data required to develop these.

\section{Conclusion}

Chronic NCDs, in particular CVD, will impose a great burden on the education system in Cape Town and possibly in the rest of SA in the future. Establishing a cohort study among teachers to measure their exposure to risk factors for NCDs, as well as the incidence of these diseases over time, will provide the critical information needed to plan prevention strategies. Implementing such a project among teachers countrywide will require the support of management structures within the education system, direct engagement with teachers and significant funding from local and international sources.

Acknowledgements. We gratefully acknowledge the PaCT Steering Committee of the Faculty of Medicine and Health Sciences at Stellenbosch University for guidance with the SA pilot study. We also thank the primary collaborators of the Africa/HSPH PaCT for their efforts in initiating, carrying out and coordinating this study in five sites (other than in SA): Clement Adebamowo (Nigeria site), Marina Njelekela (Tanzania site), David Guwatudde (Makerere, Uganda site), Francis Bajunirwe (Mbarara, Uganda site), Hans-Olov Adami and Todd Reid (HSPH co-ordinating centre). The SA pilot study was made possible through funding obtained from the Dean's office of Harvard School of Public Health (HSPH) and the HSPH Department of Nutrition, the Faculty of Medicine and Health Sciences at Stellenbosch University, and the International Society of Nephrology's Global Outreach Clinical Research and Prevention Program.

1. Mendis S, Alwan A, eds. Prioritized Research Agenda for Prevention and Control of Noncommunicable Diseases. Geneva: World Health Organization Press, 2011:25-26.

2. World Health Organization. Projected Deaths for 2005,2015 and 2030 by WHO Region Under the Baseline Scenario. Geneva: WHO, 2006. http://www.who.int/healthinfo/global_burden_disease/ Baseline Scenario. Geneva: WHO, 2006. h).
projections2002/en/ (accessed 1 April 2016).

3. Kengne AP, Ntyintyane LM, Mayosi BM. A systematic overview of prospective cohort studies of Kengne AP, Ntyintyane LM, Mayosi BM. A systematic overview of prospective cohort studies of
cardiovascular disease in sub-Saharan Africa. Cardiovasc J Afr 2012;23(2):103-112. DOI:10.5830/ Cardiovascular d

4. Dalal S, Beunza JJ, Volmink J, et al. Non-communicable diseases in sub-Saharan Africa: What we 4. Dalal S, Beunza JJ, Volmink J, et al. Non-communicable diseases in sub-S
know now. Int J Epidemiol 2011;40(4):885-901. DOI:10.1093/ije/dyr050

5. Holmes MD, Dalal S, Volmink J, et al. Non-communicable diseases in sub-Saharan Africa: The case for cohort studies. PLoS Med 2010;7(5):e1000244. DOI:10.1371/journal.pmed.1000244

6. Romieu I, Escamilla-Nunez MC, Sanchez-Zamorano LM, et al. The association between body shape silhouette and dietary pattern among Mexican women. Public Health Nutr 2012;15(1):116-125. DOI:10.1017/S1368980011001182

7. Bernstein L, Allen M, Anton-Culver H, et al. High breast cancer incidence rates among California teachers: Results from the California Teachers Study (United States). Cancer Causes Control 2002;13(7):625-635. DOI:10.1023/A:1019552126105

8. Dalal S, Holmes M, Laurence C, Bajunirwe F, Guwatudde D, Njelekel M. Feasibility of a large cohort study in sub-Saharan Africa assessed through a four-country study. Glob Health Action 2015;8:27422. DOI:10.3402/gha.v8.27422

9. Craig SR, Amin RV, Russell DW, Paradise NF. Blood cholesterol screening influence of fasting state on cholesterol results and management decisions. J Gen Intern Med 2000;15(6):395-399. state on cholesterol results and manag.

10. Mendis S. Prevention of cardiovascular disease. Pocket Guidelines for Assessment and Management of Cardiovascular Risk. WHO/ISH Cardiovascular Risk Prediction Charts. Geneva: WHO Press, 2007:5-17.

11. D’Agostino RB, Vasan RS, Pencina MJ, et al. General cardiovascular risk profile for use in primary care: The Framingham Heart Study. Circulation 2008;117(6):743-753. DOI:10.1161/ CIRCULATIONAHA.107.699579

12. Domingo AK, Asmal L, Seedat S, Esterhuizen TM, Laurence C, Volmink J. Investigating the association between diabetes mellitus, depression and psychological distress in a cohort of South African teachers. S Afr Med J 2015;105(12):1057-1060. DOI:10.7196/SAMJ.2015.v105i12.9843

13. Alwan A, Armstrong T, Bettcher D, et al. Global Status Report on Non-communicable Diseases 2010. Geneva: World Health Organization, 2010. http://www.who.int/nmh/publications/ncd report2010/en/ (accessed 3 April 2016).

14. Chow CK, Teo KK, Rangarajan S, et al. Prevalence, awareness, treatment, and control of hypertension in rural and urban communities in high-, middle-, and low-income countries. JAMA hypertension in rural and urban communities in high-,

15. Shisana O, Labadarios D, Rehle T, et al. South African National Health and Nutrition Examination Survey (SANHANES-1). Cape Town: HSRC Press, 2013:137-140. 


\section{Appendix 1. Data collection and measurements}

A core baseline questionnaire was developed in collaboration with investigators from HSPH and all African sites using the WHO STEPS instrument for surveillance of NCD risk factors as a template (http://www.who.int/chp/steps/instrument/en/index.html). The questionnaire retrospectively obtained information on demographic and lifestyle factors such as date of birth, gender, ethnicity, educational level, socioeconomic factors, tobacco use, alcohol consumption, personal and family members' medical history related to CVD and risk factors, physical activity, self-reported weight and height, and indicators of mental health.

Clinical measurements included height, weight, waist and hip circumference, blood pressure and pulse rate. Measurements were carried out by qualified nurses who underwent training, to standardise all measurements at each site. Blood pressure and pulse rate were measured according to the SA Hypertension Guidelines using an Omron 705CP-II automatic blood pressure monitor (Omron Healthcare Co., Japan) with appropriate cuff sizes (medium for an arm circumference of $22-32 \mathrm{~cm}$ and large for an arm circumference of $33-42 \mathrm{~cm}$ ). Two readings were taken 1 minute apart after a 5-minute rest period, sitting with the participant's back supported, feet resting on the floor, legs uncrossed and arm supported at heart level. If the first two readings differed by $>5 \mathrm{mmHg}$, a third reading was taken. The average of the first and second or second and third reading (when done) was taken as the blood pressure. Participants with severe hypertension ( $\geq 180$ $\mathrm{mmHg} / 110 \mathrm{mmHg}$ ) were referred to their regular doctor. Weight was determined using a Seca 813 digital floor scale (Seca GmbH \& Co, Germany), calibrated using a weight of known mass. Weight measurements were recorded to the nearest $0.1 \mathrm{~kg}$ and taken with subjects wearing minimum clothing and no shoes. Height was recorded in centimetres to one decimal using a Seca 213 stadiometer (Seca GmbH \& Co, Germany) which was placed on a hard, flat, uncarpeted surface. Readings were taken after removing any hair ornamentation and shoes, after inhalation and with the reader's eye at the level of the head piece. BMI was calculated as weight per square metre $\left(\mathrm{kg} / \mathrm{m}^{2}\right)$. Waist circumference was measured using a Seca 201 non-elastic measuring tape (Seca GmbH \& Co, Germany) placed against the skin at a horizontal plane around the midpoint between the lowest rib and the iliac crest and read at the end of normal expiration, and was recorded to the nearest $0.1 \mathrm{~cm}$. All anthropometric measurements were performed in duplicate and the average measurement used for analysis.

Random blood samples were collected to determine serum glucose, TC, HDL-C and serum creatinine. A spot urine sample was collected to determine the protein/creatinine ratio. Physical measurements and biological samples were not collected from pregnant teachers and blood glucose samples were not collected from participants who were on treatment for diabetes mellitus. Biological samples were transported daily in an icebox for processing at the Metropolis private pathology laboratory in Cape Town.

Plasma glucose was measured using an enzymatic hexokinase method. TC was determined using an enzymatic colorimetric method and HDL-C was measured directly using a homogeneous enzymatic colorimetric test. Serum and urine creatinine was determined using a kinetic colorimetric assay that is based on the Jaffé method. Urinary protein was measured using a turbidimetric method. Chemistry tests were performed on the Cobas c 501 system (Roche/Hitachi, USA). The GFR was estimated (eGFR) using the re-expressed four-variable MDRD study equation: eGFR $\left(\mathrm{mL} / \mathrm{min} / 1.73 \mathrm{~m}^{2}\right)=175 \times(($ standardised serum creatinine $\times 0.0113)-1.154) \times($ age -0.203$) \times \mathrm{F}$, where $\mathrm{F}=1$ if male and $\mathrm{F}=0.742$ if female. 